\title{
OBSERVATIONS OF THE CORONAL NETWORK
}

\author{
GEORGE W. SIMON \\ Sacramento Peak Observatory; Sunspot, N. Mex. 88349, U.S.A. \\ and \\ ROBERT W. NOYES \\ Harvard College Observatory, Cambridge, Mass. 02138, U.S.A.
}

\begin{abstract}
Spectroheliograms of quiet regions obtained by the OSO-G satellite in chromospheric, transition zone, and coronal lines indicate that the chromospheric network as seen in $\mathrm{Ca} \mathrm{K}$ can be resolved despite the low resolution ( 30 arc s) of OSO-G. The network pattern becomes more diffuse with increasing height, indicating that the magnetic field pattern spreads with height.
\end{abstract}

We wish to present a few preliminary results obtained from the Harvard experiment on the OSO-G satellite. These observations were made in a number of EUV lines
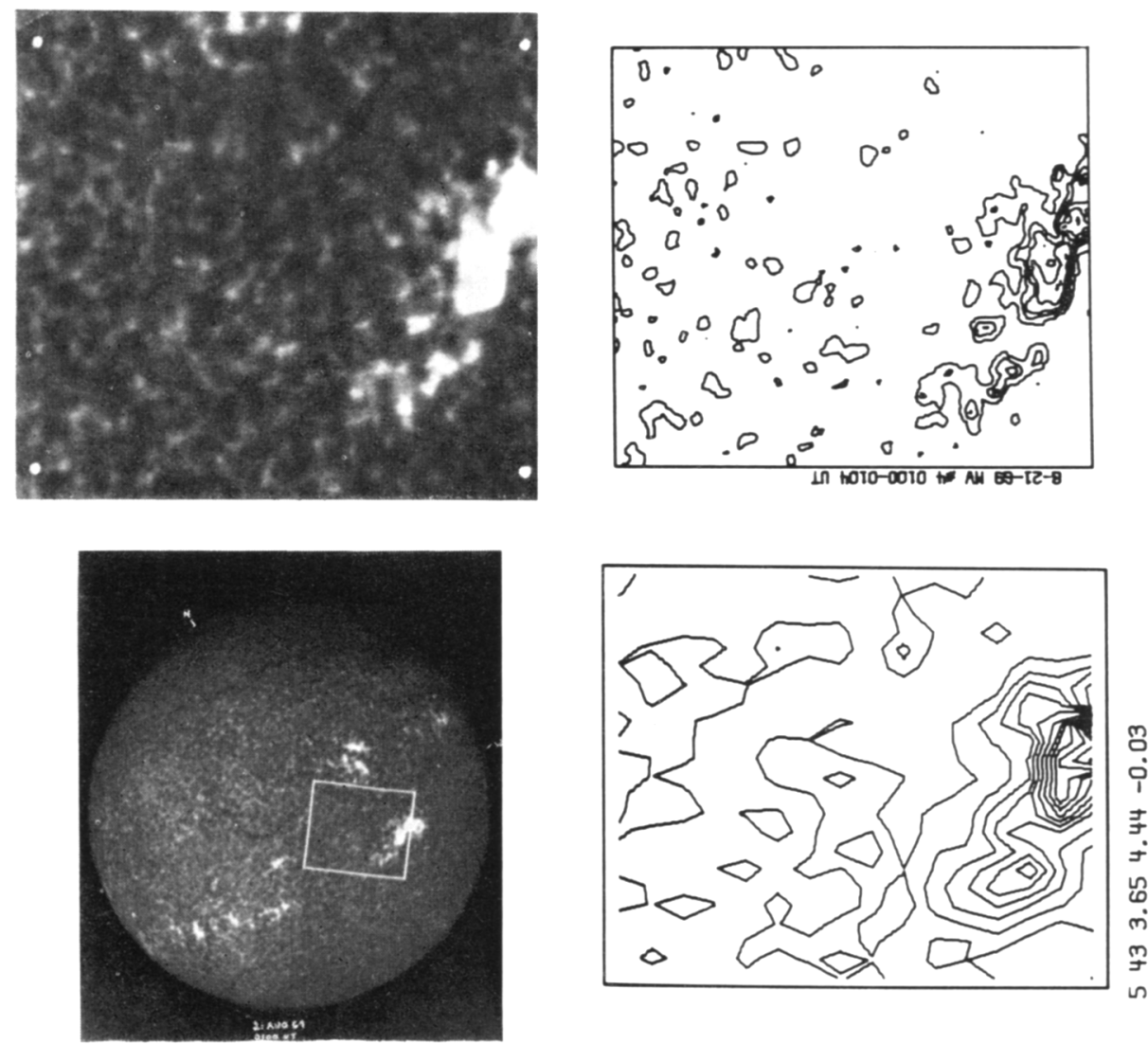

Fig. 1. Simultaneous Mt. Wilson Can 3934 and OSO-G satellite Ly Cont 897 spectroheliograms of 21 August 1969, 0100 UT. Lower left: Mt. Wilson spectroheliogram showing area observed by OSO-G. Upper left: Enlargement in Ca II of observed area. Upper right: Contour map of Ca II spectroheliogram. Lower right: Contour map of OSO-G spectroheliogram. 

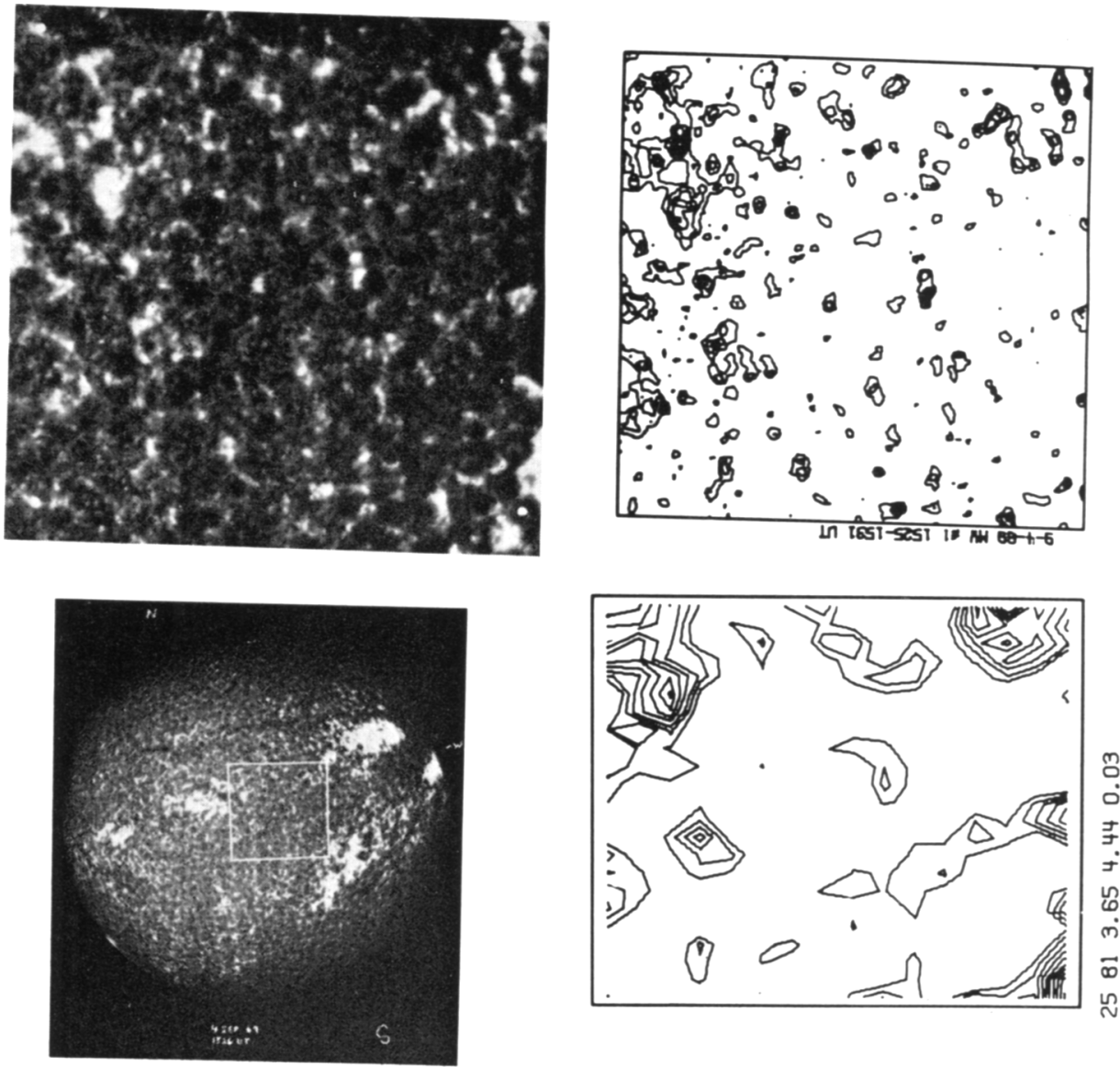

Fig. 2. Similar to Figure 1, taken on 4 September 1969, 1526 UT. In this case, the OSO-G data are of CII 1335.

which are formed between 2000 and $15000 \mathrm{~km}$ above the photosphere, that is, from the high chromosphere, through the transition zone, into the low corona. Since it is well-known that there exists a strong spatial correlation between photospheric magnetic fields and the chromospheric calcium $\mathrm{K}$ brightness network, it was hoped that these higher temperature observations would enable us to extend this correlation upward into the corona. However, there exist two difficulties. First, the photon statistics in the coronal lines were not sufficient to draw convincing conclusions, so that we were able only to go to the height of oxygen VI, which is formed in the transition zone at a temperature of $300000 \mathrm{~K}, 5000 \mathrm{~km}$ above the photosphere, that is, about $3000 \mathrm{~km}$ above calcium $\mathrm{K}$. Second, we were trying to observe the quiet-sun network pattern which has a typical scale of $30000 \mathrm{~km}$, using a telescope with an entrance aperture almost as large, namely $22000 \mathrm{~km}$ ( $30 \mathrm{arcs}$ ) on a side. Thus there were doubts whether the network structure could even be resolved with such a large aperture. 

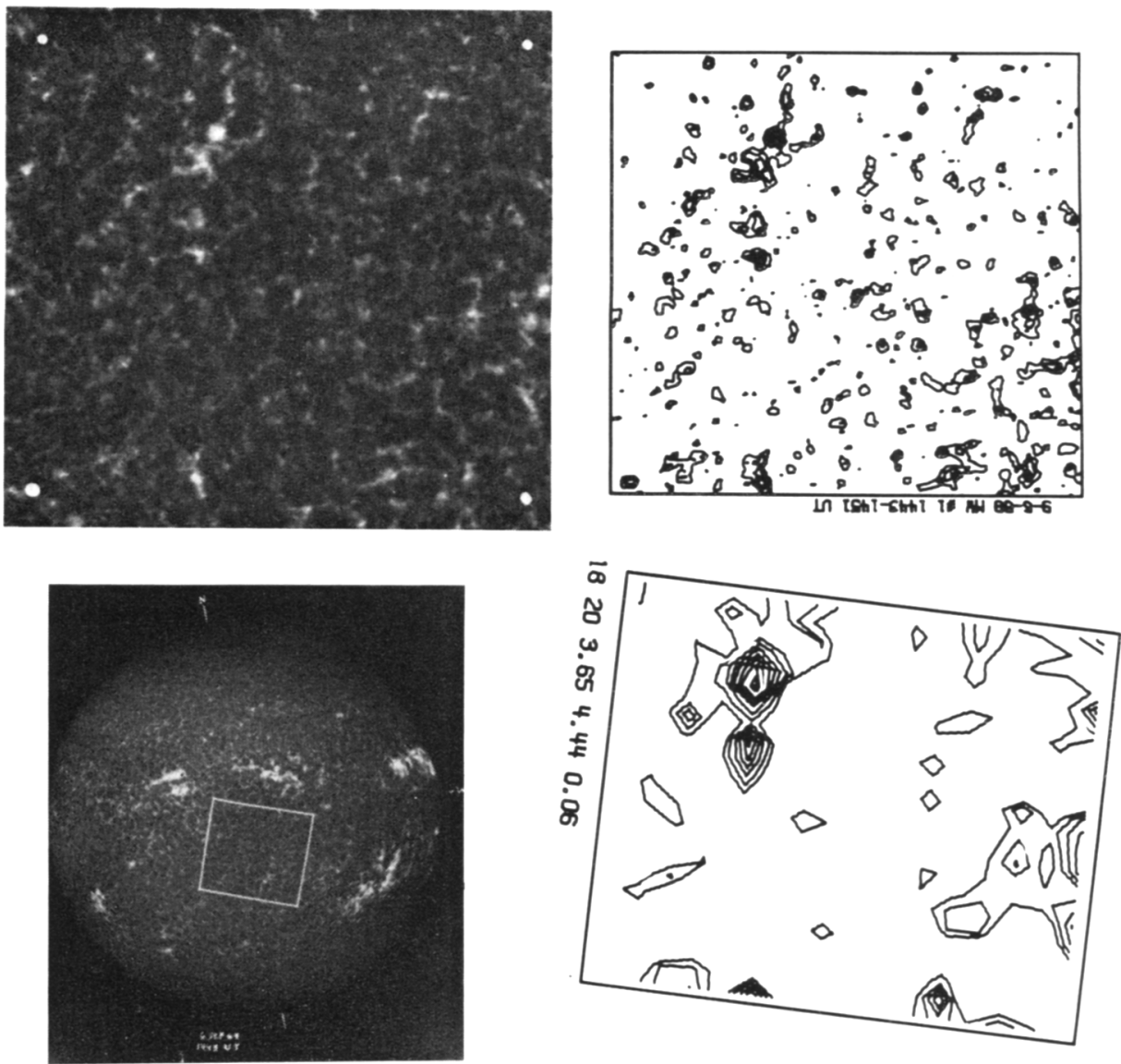

Fig. 3. Similar to Figure 1, taken on 6 September 1969, 1445 UT, with the OSO-G data in the line CII 1335.

Keeping this in mind, we will now show you some qualitative results. The lower left hand part of Figure 1 shows a calcium spectroheliogram taken at Mt. Wilson simultaneously with the satellite observation. (We wish to thank Dr. Robert Howard and his staff for their cooperation in this program.) The marked area shows the size of the satellite raster, which is $300000 \mathrm{~km}$ on a side. In the upper left you see an enlargement of the pertinent area of the calcium spectroheliogram, to the right of it a contour map of the spectroheliogram with 3 arc $s$ resolution, and at the bottom right a contour map of the satellite observation, in this case obtained in the Lyman continuum at $897 \AA$, which comes from a height very close to that of the calcium K. Note that many of the bright points in the calcium do indeed appear in the satellite observation, despite the low resolution. This correlation shows up even more clearly, if the two contour maps are superimposed, rather than shown side by side as in this figure. Two more such examples are shown in Figures 2 and 3.

On a quantitative basis, we have obtained auto-correlation curves in a number of 
these EUV lines, and the preliminary results indicate that the widths of the emission features increase with increasing height up to a width of $17000 \mathrm{~km}$ at a height of $5000 \mathrm{~km}$. We thus have the following picture of the network, and thus hopefully also of the magnetic pattern: In the photosphere we find widths under $3000 \mathrm{~km}$; in $\mathrm{H} \alpha$ at a height of $1000 \mathrm{~km}$ we find $5000 \mathrm{~km}$ widths; in calcium $\mathrm{K}$ at $2000 \mathrm{~km}$ height, the width is $10000 \mathrm{~km}$; and in oxygen VI at $5000 \mathrm{~km}$ height, the width is $17000 \mathrm{~km}$.

We conclude that, as one might expect, the magnetic field continues to spread out with increasing height, and will at some still greater height in the corona, merge together to form a more or less continuous magnetic sheath covering the solar surface. 